\title{
Short-Term Assessment of Intravitreal Dexamethasone Implant Using Enhanced-Depth Image Optical Coherence Tomography and Optical Coherence Tomography Angiography in Patients with Retinal Vascular Diseases
}

\author{
Angelo Maria Minnella • Matteo Federici • Valeria Pagliei · Angela Lanza · Gloria Gambini • \\ Carmela Grazia Caputo · Benedetto Falsini · Aldo Caporossi
}

Received: September 27, 2018 / Published online: December 18, 2018

(c) The Author(s) 2018

\begin{abstract}
Introduction: To evaluate the short-term efficacy and safety of intravitreal dexamethasone implant (IDI) in patients with macular oedema associated with diabetic retinopathy (DR) and retinal vein occlusion (RVO) using enhanceddepth image optical coherence tomography (EDI-OCT) and to estimate the effect of dexamethasone on the choroid and the retinal vascular network using OCT angiography (OCTA).
\end{abstract}

Methods: Fifteen eyes in 15 patients with macular oedema secondary to diabetes (DR, $n=8$ ) or retinal vein occlusion (RVO, $n=7$ )

Enhanced Digital Features To view enhanced digital features go to: https://doi.org/10.6084/m9.figshare. 7309151 .

A. M. Minnella $(\bowtie) \cdot$ V. Pagliei · G. Gambini ·

B. Falsini · A. Caporossi

Institute of Ophthalmology, Università Cattolica del

Sacro Cuore, Rome, Italy

e-mail: aminnella59@gmail.com

A. M. Minnella · C. G. Caputo - B. Falsini ·

A. Caporossi

Fondazione Policlinico Universitario A. Gemelli

IRCCS, Rome, Italy

M. Federici

Department of Ophthalmology, Bambino Gesù

IRCCS Children's Hospital, Rome, Italy

A. Lanza

Ospedale Cristo Re, Rome, Italy were treated with intravitreal injection of sustained-release IDI. Primary efficacy end points were changes in best corrected visual acuity and central macular thickness (CMT). Secondary end points were changes in choroidal thickness and choroidal and retinal vascular networks as determined by OCTA.

Results: CMT was significantly reduced from baseline by $3 \mathrm{~h}$ after injection $(p<0.01)$ and improved further during the 3-month followup. Visual acuity improvement was consistent with CMT reduction. No alterations in IOP or systemic side effects were observed. OCTA showed improvement from baseline in terms of decreased number and size of cysts and restoration of the retinal vascular network; flow choroidal thickness did not change significantly. CMT and visual acuity variations were similar in the two groups.

Conclusions: CMT reduced as early as $3 \mathrm{~h}$ after the injection of IDI, with further reduction during follow-up. Choroidal thickness was unchanged, whereas the vascular retinal network improved from baseline to the end of study. Both EDI-OCT and OCTA were useful in demonstrating the early beneficial effects of IDI on the macula and the perifoveal vascular network.

Funding: The article processing charges, the open access fee and the medical writing and editorial assistance was funded by Allergan. 
Keywords: Central retinal thickness; Diabetic macular oedema; Enhanced-depth image optical coherence tomography; Innovative biotechnology; Macular oedema; Ophthalmology; OCT angiography; Retinal vein occlusion; Spectral domain OCT; Sustained release intravitreal dexamethasone implant

\section{INTRODUCTION}

In the last 15 years, intravitreal pharmacotherapy has changed the therapeutic options for macular oedema (ME) related to retinal metabolic and vascular pathologies, mainly diabetic macular oedema (DME) [1] and retinal vein occlusion (RVO) [2, 3]. Diabetic retinopathy (DR) affects $1.9 \%$ of patients with diabetes mellitus [4] in whom DME is the most important cause of visual loss. Macular oedema is a consequence of DR and is due to the rupture of the blood-retinal barrier caused by a range of metabolic changes brought about by hyperglycaemia [5]. RVO is a different vascular pathology in which the sudden obstruction of a retinal vessel leads to visual loss. There are two types of RVO. Branch retinal vein occlusion (BRVO) is the most common $(0.6-1.1 \%)$ followed by central retinal vein occlusion (CRVO) $(0.1-0.4 \%)$. Both BRVO and CRVO are frequently associated with $\mathrm{ME}$, which causes visual loss. The pathogenic mechanism of ME in eyes presenting with $\mathrm{RVO}$ is multifactorial and complex. It includes the increase of venous pressure, endothelial cell dysregulation, reduction in macular vascular flow, and the spillage of many inflammatory cytokines. The pathogenesis of ME in RVO and in diabetic patients is not completely understood, but it is certain that inflammation is at least partly responsible [6, 7]. Several treatments for ME have been developed, ranging from laser treatment [8] to, more recently, the administration of intravitreal drugs such as anti-vascular endothelial growth factor (VEGF) [9] and steroids [10]. Treatment options for ME caused by BRVO include laser photocoagulation [11], intravitreal VEGF antagonists [12], and intravitreal corticosteroids [10]. Intravitreal dexamethasone implant (IDI) $0.7 \mathrm{mg}$ (Ozurdex ${ }^{\mathrm{TM}}$; Allergan, Irvine, CA, USA) has been used to reduce ME in patients with RVO and DME [10]. The long-term efficacy of this device has been demonstrated in many different studies $[13,14]$.

Optical coherence tomography (OCT) is a non-invasive imaging modality that, through cross-sectional images of the retina, allows the detection of changes in the retinal structure of eyes affected by various macular diseases and the measurement of retinal thickness [15]. In eyes with RVO and DME, OCT can display intraretinal cystoid spaces, which are responsible for the increase in retinal thickness often associated with serous detachment of the neurosensory retina. It has been demonstrated that visual function and recovery are correlated with macular thickness [16]. Furthermore, the integrity of retinal layers is the main prognostic factor linked to visual acuity and response to treatment [17]. Optical coherence tomography angiography (OCTA) is a new tool of investigation based on the principle of detecting temporal fluctuations of the OCT signal (decorrelation analysis) caused by the flow of erythrocytes and other particles in the blood vessels $[18,19]$. It provides a new technique for the visualisation of the retinal microvasculature. This tool allows the construction of threedimensional (3D) blood flow information and therefore can serve as a non-invasive method to evaluate retinal circulation. This technique does not require the use of an intravenous dye, reducing the risk of allergic reactions and other side effects, including anaphylaxis [20].

The aim of this study was to assess the early efficacy and safety of IDI in eyes affected by ME secondary to DR and RVO using enhanceddepth image OCT (EDI-OCT). This study was also intended to evaluate the effect of dexamethasone on choroidal morphology and to explore retinal vascular networks using OCTA.

\section{METHODS}

Adult patients ( $>18$ years old) with ME associated with DR and RVO were recruited from April to August 2016. The study was performed at the Institute of Ophthalmology of Università Cattolica del Sacro Cuore Fondazione Policlinico Universitario A. Gemelli-IRCCS in Rome. All 
procedures performed in studies involving human participants were in accordance with the E.C. of the Università Cattolica del Sacro Cuore; Fondazione Policlinico Universitario A. Gemelli, IRCCS, Rome Italy (prot. 53671/17, ID 1832) and with the 1964 Helsinki Declaration and its later amendments or comparable ethical standards. Informed consent was obtained from all individual participants included in the study.

All patients diagnosed with DR or RVO who developed ME with central macular thickness $(\mathrm{CMT})>250 \mu \mathrm{m}$ as measured using an OCT device were suitable to be included in the study. Patients with a history of glaucoma, high intraocular pressure (IOP), defined as IOP $>21$ mmHG, and associated ocular disease, as well as patients who received additional treatment (either a dexamethasone device or anti-VEGF injection) within 6 months preceding the enrolment visit, were excluded. Primary end points of the study were: changes in best corrected visual acuity (BCVA) and CMT as well as ocular and/or systemic side effects. Secondary end points were changes in choroidal thickness (CT) assessed using EDI-OCT examination and any difference in the vascular networks as determined analysing OCTA scans. Best corrected visual acuity, EDIOCT and IOP measurement were performed at baseline, 3 and $24 \mathrm{~h}, 1$ week, 1 month, and 3 months after IDI had been administered. The EDI-OCT analysis was performed using Heidelberg Spectralis Spectral Domain OCT (Heidelberg Engineering, Heidelberg, Germany). CMT was manually assessed on horizontal B-scans as well as $\mathrm{CT}$, which was measured from the posterior edge of the retinal pigment epithelium to the choroid-sclera junction, considering $500 \mu \mathrm{m}$ apart from the fovea. Early Treatment Diabetic Retinopathy Study (ETDRS) charts were used to assess BCVA, while a Goldmann tonometer was used to measure intraocular pressure (IOP). A 3D scan (from the outer limit of the retinal pigment epithelium to the choroid-scleral junction) was used to measure CT. All OCT images were acquired in the morning.

All patients from both DME and RVO groups underwent OCTA examination at baseline, 1 day, 7 days, and 90 days after intravitreal injection of IDI. $3 \mathrm{~mm} \times 3 \mathrm{~mm}$, $4.5 \mathrm{~mm} \times 4.5 \mathrm{~mm}$, and $6 \mathrm{~mm} \times 6 \mathrm{~mm}$ scans were acquired using Topcon's DRI Triton sweptsource OCT device and best quality images were retained for further qualitative analysis. All the selected scans were examined by two independent investigators. The statistical analyses were performed by a biostatistician and were conducted with SPSS Statistics for Windows, version 22.0 (IBM Corp, Armonk, NY, USA). The variations in CMT from baseline to the end of the follow-up period were analysed with repeatedmeasures ANOVA tests $(p<0.001$; standard error $=38.25 ; F(5.93)=21.64)$.

\section{RESULTS}

A total of 15 eyes of 15 patients were evaluated, and all of them were treated with intravitreal implantation of the IDI. Six patients were injected for the first time (naïve to treatment). The average age was $68.7 \pm 12.4$ years; 7 of 15 patients were male (Table 1 ). Of the 15 eyes

Table 1 Baseline patient characteristics by sex, age, diagnosis, previous treatments, and lens status

\begin{tabular}{llllll}
\hline Patient & Sex & Age, years & Diagnosis & Naive & Phakic \\
\hline 1 & F & 73 & RVO & No & Yes \\
2 & M & 57 & RVO & Yes & Yes \\
3 & M & 57 & DME & Yes & Yes \\
4 & F & 68 & RVO & No & Yes \\
5 & M & 55 & DME & Yes & Yes \\
6 & F & 51 & DME & No & Yes \\
7 & F & 48 & RVO & No & Yes \\
8 & M & 69 & DME & No & Yes \\
9 & F & 79 & DME & No & Yes \\
10 & F & 81 & DME & Yes & No \\
11 & M & 77 & RVO & Yes & No \\
12 & M & 52 & RVO & Yes & Yes \\
13 & F & 84 & RVO & No & Yes \\
14 & M & 66 & DME & No & Yes \\
15 & F & 69 & DME & No & Yes \\
\hline
\end{tabular}

$D M E$ diabetic macular oedema, $R V O$ retinal vein occlusion, $N A \ddot{I} V E$ naïve to treatment 
examined, 8 had DME and 7 had RVO (4 were affected by CRVO and 3 by BRVO).

Before the treatment, no large difference was observed within each of the two groups of patients (DME and RVO) regarding mean age, sex, duration of diabetes, level of glycated haemoglobin, level of arterial hypertension, or time since onset of RVO. Two patients ( 2 eyes) of 15 were pseudophakic; 13 patients (13 eyes) were phakic at the time of enrolment. None showed cataract progression during the 3-month followup period.

The mean CMT before injection was $480.6 \pm 143.2 \mu \mathrm{m}$. A reduction in CMT $(402.8$ $\mu \mathrm{m} \pm 152.7)$ with a mean reduction of $77.8 \mu \mathrm{m}$ was observed in all the eyes as soon as $3 \mathrm{~h}$ after the treatment (Figs. 1, 2). The decrease in CMT was progressive, as mean CMT was $296.7 \pm 109.9 \mu \mathrm{m} 3$ months after the administration of the drug (Figs. 1, 2, 3; Table 2). Decreased visual acuity was found $3 \mathrm{~h}$ (T1) after IDI was injected. In contrast, an improvement in visual acuity was observed $24 \mathrm{~h}$ after treatment, consistent with the CMT reduction. The trend of responses (in terms of CMT) among the different subgroups (DME naive, DME previously treated; RVO naïve, RVO previously treated) was analysed as well. Considering the small sample size, these considerations are not

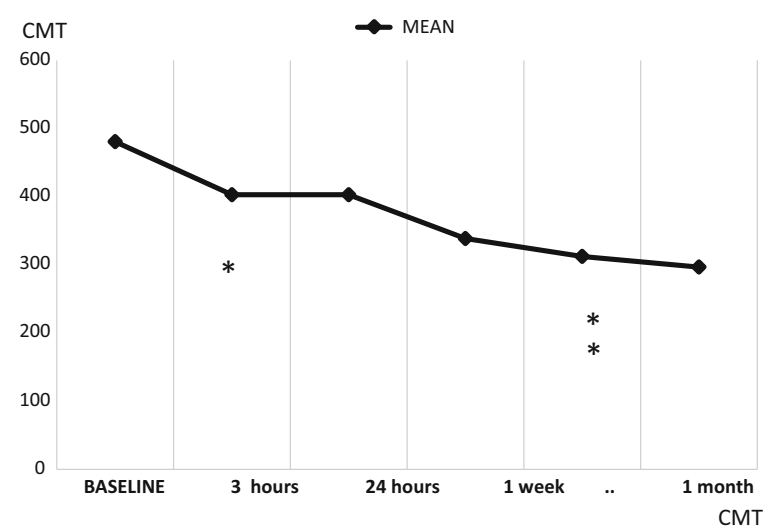

Fig. 1 Graph showing the linear regression of central macular thickness (CMT) as a function of time. Analysis of the early effect of dexamethasone from $3 \mathrm{~h}$ to 3 months after intravitreal injection. The single asterisk is related to a $P$ value $<0.05$, whereas the double asterisk corresponds to a $P$ value $<0.001$, representing the maximum level of statistical significance

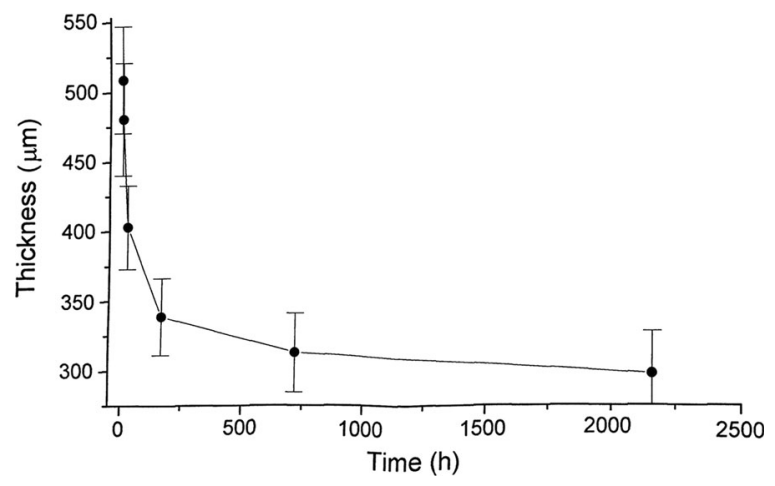

Fig. 2 Figure showing the trend of action of the drug as a function of time. Specifically, the reduction of central macular thickness is statistically significant after $3 \mathrm{~h}$, and it continues after $80 \mathrm{~h}$, reaching the half-life of the drug

statistically representative. At the end of the follow-up period, a decrease in CMT was found in all the subgroups. Comparing the DME subgroups (DME naive, DME previously treated), the difference in CMT values between T5 (3 months after the injection) and the baseline (T0) was greater in the DME previously treated subgroup. Regarding the RVO subgroups, the reduction in CMT at the end of the follow-up period was greater in the naive subgroup than in the previously treated one. Analysing the trend of the curve showing the progression of the CMT values, the decrease was more gradual in the DME subgroups. Contrarily, the graph appeared to be steeper, especially at the beginning of the follow-up period, in the RVO subgroups, particularly in the naïve one. No changes were observed in CT.

The mean best corrected visual acuity (BCVA) in the study eyes (expressed in ETDRS letters) was: $36.4 \pm 9.5$ letters at T0 (baseline), $35.4 \pm 9.1$ at $\mathrm{T} 1(3 \mathrm{~h}), 36.1 \pm 10.6$ at T2 $(24 \mathrm{~h})$, $39.8 \pm 9.06$ at T3 (1 week), $41.9 \pm 9.6$ at T4 (1 month), and $45.4 \pm 10.1$ letters at T5 (3 months).

The mean IOP in study eyes (expressed in $\mathrm{mmHg}$ ) was: $15.3 \pm 2.5 \mathrm{mmHg}$ at baseline (T0), $18.4 \pm 3.4$ at $\mathrm{T} 1(3 \mathrm{~h}), 17.3 \pm 3.1$ at $\mathrm{T} 2(24 \mathrm{~h})$, $17.06 \pm 3.5$ at T3 (1 week), $16.6 \pm 3.09$ at T4 (1 month), and $16.4 \pm 2.9 \mathrm{mmHg}$ at $\mathrm{T} 5$ (3 months).

On analysing the OCTA scans, an early improvement from baseline was observed in 


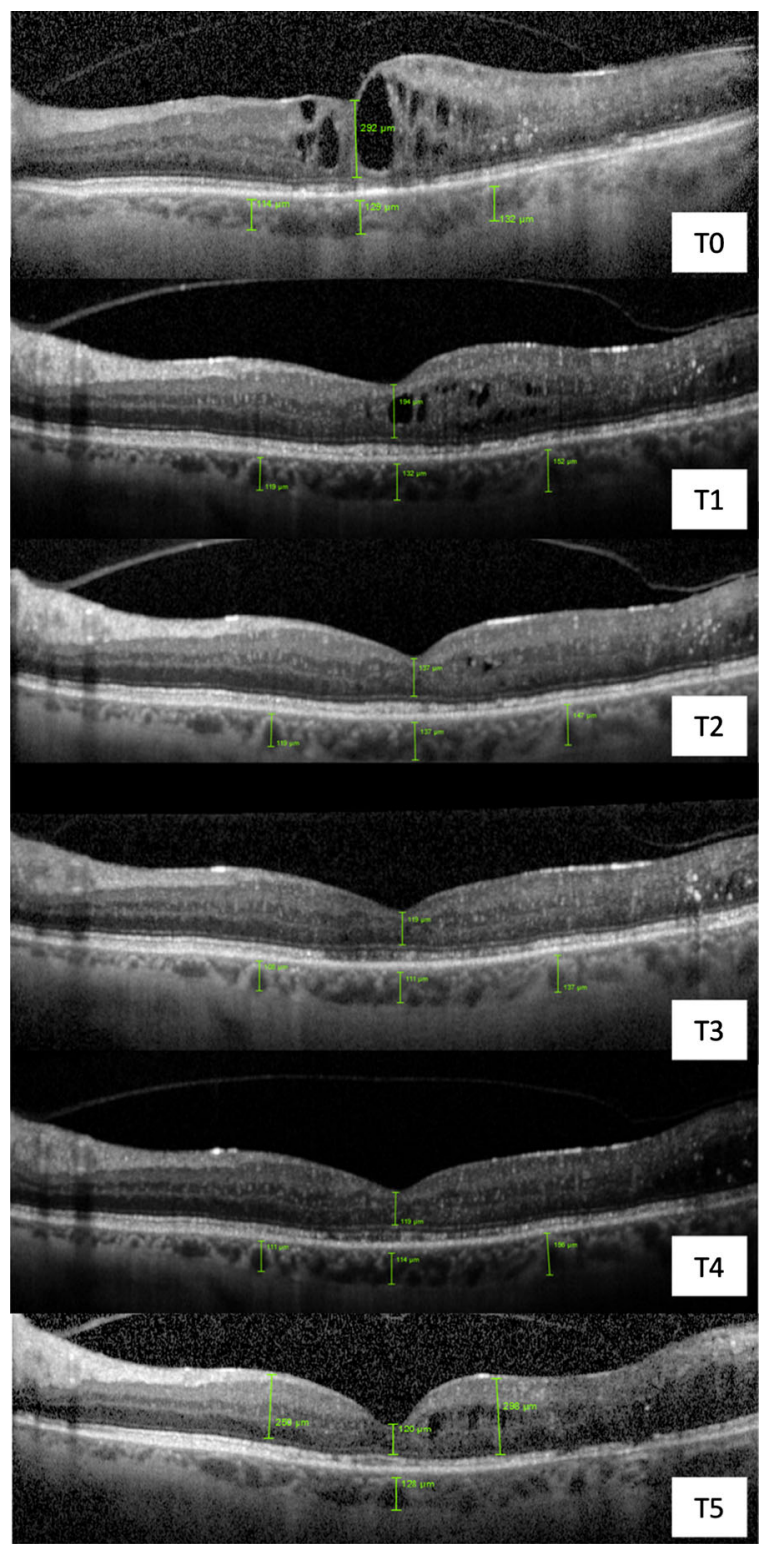

Fig. 3 Example of an enhanced-depth image-optical coherence tomography (EDI-OCT) of a diabetic macular oedema eye in a patient (\#5) who underwent an intravitreal injection of long-release dexamethasone $700 \mu \mathrm{g}$ at baseline (T0). The sequences of the structural OCT B-scan represent the timing of follow-up starting from $3 \mathrm{~h}$ (T1) to 3 months (5). T2 = $24 \mathrm{~h}$; T3 = 1 week; $\mathrm{T} 4=1$ month

both the DME and RVO groups. In eyes affected by DME, the deep retinal capillary plexus was mainly involved. Dark spaces corresponding to cystoid macular oedema and microvascular remodelling (microaneurysms) were noticed during baseline evaluation. In the majority of cases, the images obtained 1 day after the administration of the drug showed a decrease in the number and size of the cysts. A reduction in the number of microaneurysms and a partial vascular restoration was more evident a week after the injection and at the end of the followup period (Fig. 4).

In eyes affected by RVO, pathological features were noticed in both the superficial and deep retinal capillary plexuses. Perifoveal ischaemic areas and disruption of the foveal capillary arcade were observed in the superficial capillary network, while cystic spaces were visible in scans performed at the level of the deep capillary plexus. Tortuous and dilated vessels could be seen in both the superficial and deep retinal vascular networks, but mainly in the superficial one. A reduction in the number and size of the cysts was seen 1 day after the injection. Vascular restoration and decreased dilatation and tortuosity of the vessels were more evident on OCTA images obtained 1 week after IDI insertion (Fig. 5).

No significant difference in IOP was found at the end of the study. None of the patients in our study had any systemic side effects. Two patients had a local adverse event, a corneal abrasion after injection and a temporary increase in IOP $(24 \mathrm{mmHg})$, which was treated with local drops. Both complications resolved.

\section{DISCUSSION}

Diabetic retinopathy and RVO are prominent diseases worldwide and represent the most common retinal vascular disorders, with $\mathrm{ME}$ being the main cause of visual impairment. Although the pathogenesis of ME appears to be multifactorial, inflammatory mediators play a key role [21]. Several studies on the analysis of aqueous humour in patients with ME have shown an increase in cytokines and inflammatory factors $[22,23]$. Therefore, corticosteroids can be useful against the inflammatory components of ME [24]. 
Table 2 Central macular thickness (CMT) change (in microns) as a function of time

\begin{tabular}{lllllll}
\hline Patient & Baseline T0 & $\mathbf{3} \mathbf{h}, \mathbf{T 1}$ & $\mathbf{2 4} \mathbf{~ h , ~ T 2}$ & $\mathbf{1}$ week, T3 & $\mathbf{1}$ month, T4 & $\mathbf{3}$ months, T5 \\
\hline 1 & 412 & 407 & 310 & 293 & 277 & 267 \\
2 & 258 & 238 & 244 & 252 & 300 & 330 \\
3 & 727 & 769 & 557 & 387 & 365 & 377 \\
4 & 407 & 368 & 367 & 147 & 122 & 108 \\
5 & 358 & 313 & 277 & 250 & 222 & 234 \\
6 & 487 & 496 & 418 & 402 & 333 & 377 \\
7 & 555 & 533 & 391 & 341 & 346 & 234 \\
8 & 299 & 282 & 327 & 333 & 322 & 311 \\
9 & 706 & 661 & 619 & 611 & 606 & 599 \\
10 & 465 & 446 & 422 & 422 & 402 & 392 \\
11 & 644 & 559 & 331 & 305 & 275 & 244 \\
12 & 564 & 535 & 338 & 322 & 226 & 221 \\
13 & 443 & 391 & 366 & 301 & 299 & 233 \\
14 & 575 & 480 & 456 & 273 & 222 & 202 \\
15 & 701 & 731 & 620 & 441 & 368 & 322 \\
Mean & 480.6 & 402.8 & 402.8 & 338.6 & 312.3 & 296.7 \\
Standard deviation & 143.2 & 152.7 & 112.1 & 103.1 & 104.8 & 109.9 \\
\hline
\end{tabular}

The values reported in the figure were manually obtained, whereas those reported in Table 2 were directly provided by the instrument

The study was intended to evaluate the reduction in CMT after $3 \mathrm{~h}, 24 \mathrm{~h}, 1$ week, 1 month, and 3 months after IDI to highlight the early (and long-term) effects of dexamethasone implants. Our findings of a significant reduction in CMT after $3 \mathrm{~h}$ support the hypothesis that intravitreal long-release steroids play a role in humans, inducing a rapid response in terms of reduction of CMT. Veritti investigated the effect of dexamethasone implants on macular morphology and visual function in eyes with RVO [25] and DME [26]. In both pathologies, a fast reduction in macular thickness was seen, starting 1 day after the administration of the drug. Recently, Lo Giudice investigated the regression of diabetic macular oedema in the first $3 \mathrm{~h}$ after the injection, showing the early effect of IDI [27].
Regarding the efficacy of dexamethasone on DME and especially the earliness of its effect, our data are similar to those reported in this study. Furthermore, in our study, the effect of intravitreally administrated steroids was also observed in eyes with RVO.

The anatomical data relating to reduction in CMT are consistent with functional improvement. In our opinion, the initial visual acuity impairment ( $3 \mathrm{~h}$ post-injection) is likely to be related to the injection procedure rather than permanent retinal damage. Preparatory drops of local anaesthetic and povidone iodine 10\% conjunctival sac irrigation might have caused patient discomfort and difficulties in performing a BCVA test. The subsequent improvement in BCVA related to the decrease in retinal thickness confirms the transitory nature of the 


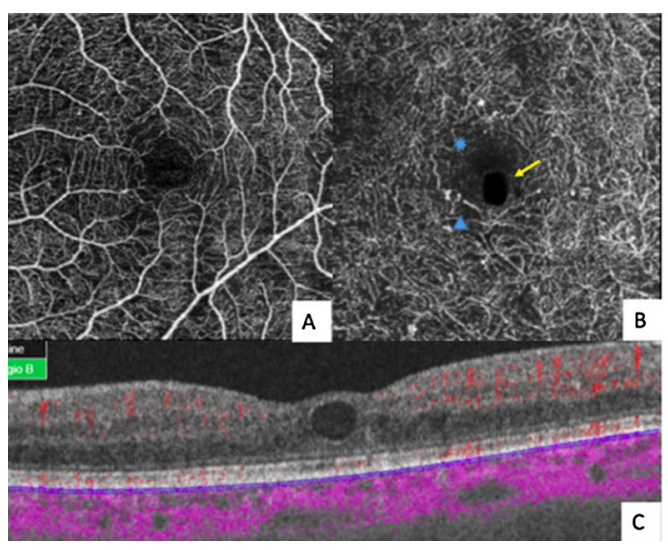

Fig. 4 Figure showing the modification of the superficial and deep capillary plexuses of patient \#10 at baseline (T0) and T5 (3 months after dexamethasone injection). In the deep network, the reduction of the central macular no-flow aspect connected to cystic space (arrow) is shown together

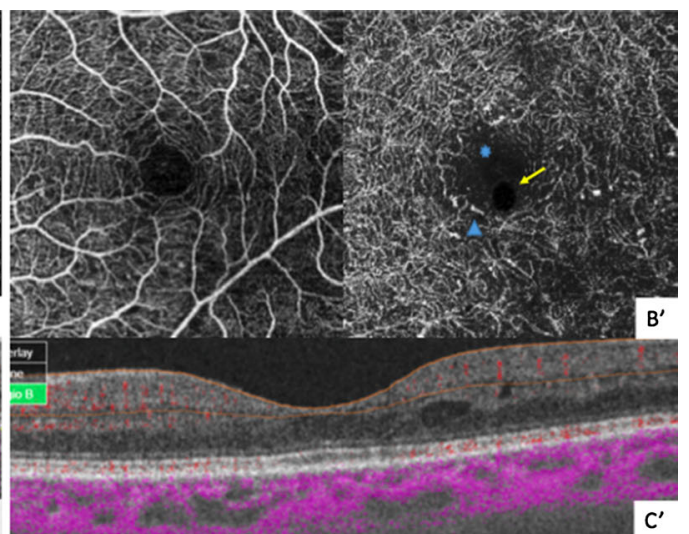

with the decrease of the no-ischaemic area (asterisk) regarding the foveal avascular zone (FAZ) and the remodelling of the hyperreflective spot connected to microaneurysm (arrowhead). The reduction of central macular cysts is shown in $\mathrm{C}^{\prime}$
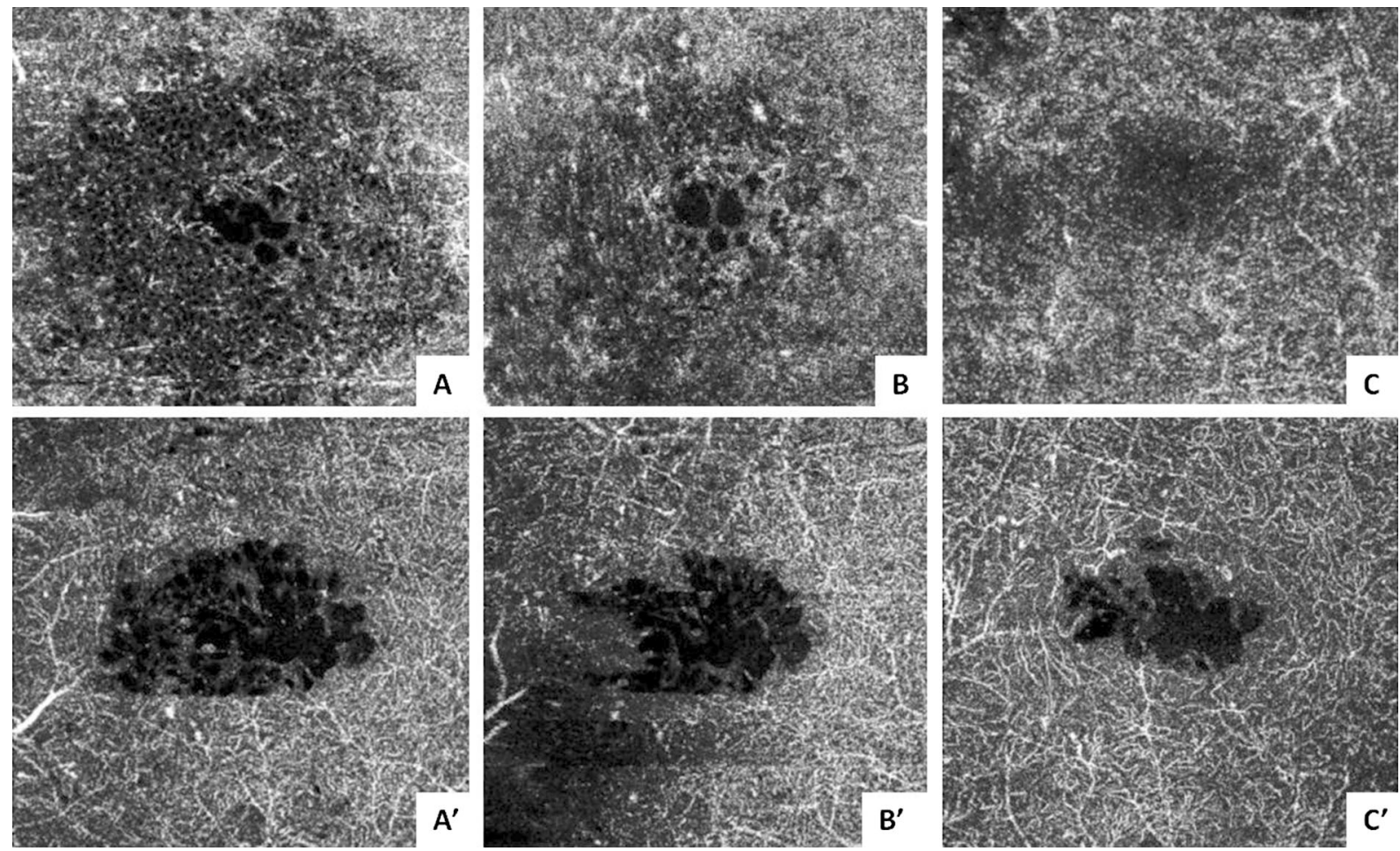

Fig. 5 Optical coherence tomography angiography (OCTA) showing the effects of intravitreal dexamethasone implant (IDI) on retinal vascularisation in the eyes of two patients with diabetic macular oedema. Cystoid macular oedema and microaneurysms can be seen on OCTA images of the deep capillary plexus, obtained during the baseline examination $\left(\mathbf{a}, \mathbf{a}^{\prime}\right)$. OCTA scans acquired 1 day after the injection $\left(\mathbf{b}, \mathbf{b}^{\prime}\right)$ show a reduction in the number and size of the cysts. The decrease progresses further and is even more evident 1 week after the administration of IDI $\left(\mathbf{c}, \mathbf{c}^{\prime}\right)$ concurrently with a partial vascular restoration 
earlier disability. Considering these early effects, we speculate that in patients affected by $\mathrm{ME}$ and cataract that need both treatments, slow-release dexamethasone injection can be performed in the same surgical setting of the cataract extraction, avoiding a double surgery where the injection precedes the cataract surgery by days or weeks.

Regarding the role of the choroid, several reports support the hypothesis that choroidal thickness is involved in the pathophysiology of many retinal diseases [28-31]. The pro-inflammatory role of the choroid in DR and DME is still controversial. Similarly, there is no agreement concerning choroidal morphology and thickness in eyes affected by RVO. A study conducted by Lee showed an increased CT in eyes with RVO with a considerable decrease after Ozurdex ${ }^{\mathrm{TM}}$ injection [31]. In our study, no significant changes in CT were detectable following IDI in either the DME or RVO group. However, the small sample size and choroidal thickness being manually measured represent important limitations.

The recent OCTA technology offers a noninvasive option to study both structural and functional characteristics of the blood flow. It provides a detailed view of the retinal vasculature and, giving the opportunity to separately investigate each capillary plexus, allows clinicians to detect any microvascular abnormalities. The above-mentioned characteristics explain the emerging key role of OCTA as a valid tool in the follow-up of patients affected by retinal vascular disease.

A study conducted by Glacet-Bernard has already investigated the changes in the superficial and deep capillary plexuses and the choriocapillaris of eyes affected by RVO [32]. In the study, OCTA images were retrospectively reviewed. On the other hand, Toto analysed OCTA scans of eyes affected by DME, with the images acquired at the baseline examination and 7, 30, 60, 90 and 120 days after dexamethasone administration [33]. In terms of qualitative improvement in both the superficial and deep capillary plexuses, our observations were comparable to those of the above-mentioned studies. Moreover, in our study, the effect of IDI on capillary plexuses was assessed through the analysis of OCTA scans obtained just 1 day after treatment. A reduction in the number and size of the cysts and in the extent of ME at the level of the deep capillary network was already visible 1 day after the injection in both the DME and the RVO groups. Despite the early onset, these changes were more evident 1 week after IDI administration and at the end of the follow-up period. This further improvement in $\mathrm{ME}$ was concurrent with the beginning of a vascular restoration mainly concerning the deep capillary plexus in the DME group and both the superficial and deep networks in eyes affected by RVO.

Since RVO and DME are both vascular diseases, we believe using OCTA is important to explore the effect of dexamethasone on the superficial and deep retinal vascular plexuses and to investigate the ability of this drug to reverse diabetic pathological features. In this perspective, this new tool can provide an additional source to display the time at which dexamethasone exerts its effect.

In conclusion, this study confirms the very early effect of IDI within the first $3 \mathrm{~h}$ from injection in both DME and RVO, persisting during the follow-up period. The fast efficacy of the implant in remodelling the macular profile may be the key to prevent degenerative and irreversible phenomena linked to the persistence of cystoid oedema in the central retina. No significant local (cataract and/or increase in IOP) or systemic side effects were noted during the study, thus confirming the short-term safety profile of slow-release intravitreal dexamethasone. The key role of multimodal imaging techniques such as EDI-OCT and OCT angiography in investigating retinal restoration has been underlined. These tools were helpful in confirming the early structural changes following IDI and consisting in a reduction of CMT and remodelling of the deep capillary plexus in DME patients and of both the superficial and deep capillary plexuses in the RVO group.

\section{ACKNOWLEDGEMENTS}

We thank the study participants. 
Funding. No funding or sponsorship was received for this study. The article processing charges, the open access fee and the medical writing and editorial assistance was funded by Allergan.

\section{Medical Writing and Editorial Assis-} tance. Writing and editorial assistance was provided to the authors by Ray Hill on behalf of Health Publishing \& Services Srl and funded by Allergan plc.

Authorship. All named authors meet the International Committee of Medical Journal Editors (ICMJE) criteria for authorship for this article, take responsibility for the integrity of the work as a whole, and have given their approval for this version to be published.

Disclosures. Angelo Maria Minnella, Matteo Federici, Valeria Pagliei, Angela Lanza, Gloria Gambini, Carmela Grazia Caputo, Benedetto Falsini, and Aldo Caporossi have nothing to disclose.

Compliance with Ethics Guidelines. All procedures performed in studies involving human participants were in accordance with the E.C. of the Università Cattolica del Sacro Cuore; Fondazione Policlinico Universitario A. Gemelli, IRCCS, Rome Italy (prot. 53671/17, ID 1832) and with the 1964 Helsinki Declaration and its later amendments or comparable ethical standards. Informed consent was obtained from all individual participants included in the study.

Data Availability. The data sets during and/ or analysed during the current study are available from the corresponding author on reasonable request.

Open Access. This article is distributed under the terms of the Creative Commons Attribution-NonCommercial 4.0 International License (http://creativecommons.org/licenses/ by-nc/4.0/), which permits any noncommercial use, distribution, and reproduction in any medium, provided you give appropriate credit to the original author(s) and the source, provide a link to the Creative Commons license, and indicate if changes were made.

\section{REFERENCES}

1. Cheung N, Mitchell P, Wong TY. Diabetic retinopathy. Lancet. 2010;376(9735):124-36.

2. Klein R, Klein BE, Moss SE, Meuer SM. The epidemiology of retinal vein occlusion: the Beaver Dam Eye Study. Trans Am Ophthalmol Soc. 2000;98:133-41.

3. Laouri M, Chen E, Looman M, Gallagher M. The burden of disease of retinal vein occlusion: review of the literature. Eye (Lond). 2011;25(8):981-8.

4. Romero-Aroca P, de la Riva-Fernandez S, Valls-Mateu A, Sagarra-Alamo R, Moreno-Ribas A, Soler N. Changes observed in diabetic retinopathy: eightyear follow-up of a Spanish population. Br J Ophthalmol. 2016;100(10):1366-71.

5. Romero-Aroca P. Targeting the pathophysiology of diabetic macular edema. Diabetes Care. 2010;33(11):2484-5.

6. Kaneda S, Miyazaki D, Sasaki S, Yakura K, Terasaka Y, Miyake K, et al. Multivariate analyses of inflammatory cytokines in eyes with branch retinal vein occlusion: relationships to bevacizumab treatment. Invest Ophthalmol Vis Sci. 2011;52(6):2982-8.

7. Romero-Aroca P, Baget-Bernaldiz M, Pareja-Rios A, Lopez-Galvez M, Navarro-Gil R, Verges R. Diabetic macular edema pathophysiology: vasogenic versus inflammatory. J Diabetes Res. 2016;2016:2156273.

8. Early Treatment Diabetic Retinopathy Study Research Group. Techniques for scatter and local photocoagulation treatment of diabetic retinopathy: early Treatment Diabetic Retinopathy Study Report no. 3. Int Ophthalmol Clin. 1987;27(4):254-64.

9. Stefanini FR, Badaro E, Falabella P, Koss M, Farah ME, Maia M. Anti-VEGF for the management of diabetic macular edema. J Immunol Res. 2014;2014:632307.

10. Haller JA, Bandello F, Belfort R Jr, Blumenkranz MS, Gillies M, Heier J, et al. Dexamethasone intravitreal implant in patients with macular edema related to branch or central retinal vein occlusion twelvemonth study results. Ophthalmology. 2011;118(12):2453-60.

11. Branch Vein Occlusion Study Group. Argon laser photocoagulation for macular edema in branch 
vein occlusion. Am J Ophthalmol. 1984;98(3):271-82.

12. Gerding H, Mones J, Tadayoni R, Boscia F, Pearce I, Priglinger S. Ranibizumab in retinal vein occlusion: treatment recommendations by an expert panel. Br J Ophthalmol. 2015;99(3):297-304.

13. Querques L, Querques G, Lattanzio R, Gigante SR, Del Turco C, Corradetti G, et al. Repeated intravitreal dexamethasone implant (Ozurdex(R)) for retinal vein occlusion. Ophthalmologica. 2013;229(1):21-5.

14. Chang-Lin JE, Attar M, Acheampong AA, Robinson MR, Whitcup SM, Kuppermann BD, et al. Pharmacokinetics and pharmacodynamics of a sustainedrelease dexamethasone intravitreal implant. Invest Ophthalmol Vis Sci. 2011;52(1):80-6.

15. Catier A, Tadayoni R, Paques M, Erginay A, Haouchine B, Gaudric A, et al. Characterization of macular edema from various etiologies by optical coherence tomography. Am J Ophthalmol. 2005;140(2):200-6.

16. Ota M, Tsujikawa A, Kita M, Miyamoto K, Sakamoto A, Yamaike N, et al. Integrity of foveal photoreceptor layer in central retinal vein occlusion. Retina. 2008;28(10):1502-8.

17. Murakami T, Yoshimura N. Structural changes in individual retinal layers in diabetic macular edema. J Diabetes Res. 2013;2013:920713.

18. Savastano MC, Lumbroso B, Rispoli M. In vivo characterization of retinal vascularization morphology using optical coherence tomography angiography. Retina. 2015;35(11):2196-203.

19. de Carlo TE, Romano A, Waheed NK, Duker JS. A review of optical coherence tomography angiography (OCTA). Int J Retina Vitreous. 2015;1(1):5.

20. Ha SO, Kim DY, Sohn CH, Lim KS. Anaphylaxis caused by intravenous fluorescein: clinical characteristics and review of literature. Intern Emerg Med. 2014;9(3):325-30.

21. Vujosevic S, Torresin T, Bini S, Convento E, Pilotto E, Parrozzani R, et al. Imaging retinal inflammatory biomarkers after intravitreal steroid and anti-VEGF treatment in diabetic macular oedema. Acta Ophthalmol. 2017;95(5):464-71.

22. Jonas JB, Jonas RA, Neumaier M, Findeisen P. Cytokine concentration in aqueous humor of eyes with diabetic macular edema. Retina. 2012;32(10):2150-7.

23. Noma H, Funatsu H, Yamasaki M, Tsukamoto H, Mimura T, Sone T, et al. Pathogenesis of macular edema with branch retinal vein occlusion and intraocular levels of vascular endothelial growth factor and interleukin-6. Am J Ophthalmol. 2005;140(2):256-61.

24. Caceres-del-Carpio J, Costa RD, Haider A, Narayanan R, Kuppermann BD. Corticosteroids: triamcinolone, Dexamethasone and Fluocinolone. Dev Ophthalmol. 2016;55:221-31.

25. Veritti D, Macor S, Lanzetta P. Early effects of dexamethasone implant on macular morphology and visual function in patients with macular edema secondary to retinal vein occlusion. Ophthalmologica. 2014;232(3):144-8.

26. Veritti D, Sarao V, Galiazzo F, Lanzetta P. Early effects of dexamethasone implant on macular morphology and visual function in patients with diabetic macular edema. Ophthalmologica. 2017;238(1-2):100-5.

27. Lo Giudice G, Avarello A, Campana G, Galan A. Rapid response to dexamethasone intravitreal implant in diabetic macular edema. Eur J Ophthalmol. 2018;28(1):74-9.

28. Balasubramanian S, Lei J, Nittala MG, Velaga SB, Haines J, Pericak-Vance MA, et al. Association of drusen volume with choroidal parameters in nonneovascular age-related macular degeneration. Retina. 2017;37(10):1880-7.

29. Campos A, Campos EJ, Martins J, Ambrosio AF, Silva R. Viewing the choroid: where we stand, challenges and contradictions in diabetic retinopathy and diabetic macular oedema. Acta Ophthalmol. 2017;95(5):446-59.

30. Kim JH, Kang SW, Kim JR, Kim SJ. Variability of subfoveal choroidal thickness measurements in patients with age-related macular degeneration and central serous chorioretinopathy. Eye (Lond). 2013;27(7):809-15.

31. Lee EK, Han JM, Hyon JY, Yu HG. Changes in choroidal thickness after intravitreal dexamethasone implant injection in retinal vein occlusion. $\mathrm{Br}$ J Ophthalmol. 2015;99(11):1543-9.

32. Glacet-Bernard A, Sellam A, Coscas F, Coscas G, Souied EH. Optical coherence tomography angiography in retinal vein occlusion treated with dexamethasone implant: a new test for follow-up evaluation. Eur J Ophthalmol. 2016;26(5):460-8.

33. Toto L, D'Aloisio R, Di Nicola M, Di Martino G, Di Staso S, Ciancaglini $M$, et al. Qualitative and quantitative assessment of vascular changes in diabetic macular edema after dexamethasone implant using optical coherence tomography angiography. Int J Mol Sci. 2017;18(6):1181. 